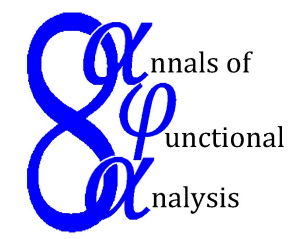

Ann. Funct. Anal. 6 (2015), no. 2, 133-142

http://doi.org/10.15352/afa/06-2-12

ISSN: 2008-8752 (electronic)

http://projecteuclid.org/afa

\title{
HIGHER RANK NUMERICAL RANGES OF RECTANGULAR MATRICES
}

\author{
MOHSEN ZAHRAEI ${ }^{1}$ AND GHOLAMREZA AGHAMOLLAEI ${ }^{2 *}$ \\ Communicated by T. Yamazaki
}

\begin{abstract}
In this paper, the notions of rank $-k$ numerical range and $k$-spectrum of rectangular complex matrices are introduced. Some algebraic and geometrical properties are investigated. Moreover, for $\epsilon>0$, the notion of Birkhoff-James approximate orthogonality sets for $\epsilon$-higher rank numerical ranges of rectangular matrices is also introduced and studied. The proposed definitions yield a natural generalization of standard higher rank numerical ranges.
\end{abstract}

\section{INTRODUCTION AND PRELIMINARIES}

Let $M_{n \times m}$ be the vector space of all $n \times m$ complex matrices. For the case $n=$ $m, M_{n \times n}$ is denoted by $M_{n}$; namely, the algebra of all $n \times n$ complex matrices. Also, $\mathcal{U}_{n}$ denotes the group of $n \times n$ unitary matrices. A quantum channel is a trace preserving completely positive map such as $L: M_{n} \longrightarrow M_{n}$. By the structure of completely positive linear maps, e.g., see [4], there are matrices $E_{1}, \ldots, E_{r} \in M_{n}$ with $\sum_{j=1}^{r} E_{j} E_{j}^{*}=I_{n}$ such that $L(A)=\sum_{j=1}^{r} E_{j}^{*} A E_{j}$. The matrices $E_{1}, \ldots, E_{r}$ are interpreted as the error operators of the quantum channel $L$.

Let $V$ be a $k$-dimensional subspace of $\mathbb{C}^{n}$ and $P$ be the orthogonal projection of $\mathbb{C}^{n}$ onto $V$. Then the $k$-dimensional subspace $V$ is a quantum error correction code for the channel $L$ if and only if there are scalars $\gamma_{i j} \in \mathbb{C}$ with $i, j \in\{1, \ldots, r\}$ such that $P E_{i}^{*} E_{j} P=\gamma_{i j} P$; for more information, see [8] and its references, and also see [11]. In this connection, for a positive integer $1 \leq k \leq n$, the rank-k numerical range of $A \in M_{n}$ is defined and denoted by

$$
\Lambda_{k}(A)=\left\{\lambda \in \mathbb{C}: P A P=\lambda P, \text { for some rank }-k \text { orthogonal projection } P \text { on } \mathbb{C}^{n}\right\} .
$$

Date: Received: Mar. 28, 2014; Revised: Jul. 8, 2014; Accepted: Jul. 22, 2014.

* Corresponding author.

2010 Mathematics Subject Classification. Primary 15A60; Secondary 47A30, 15A18, 81P68.

Key words and phrases. Rank- $k$ numerical range, isometry, numerical range, rectangular matrices, $k$-spectrum. 
By [5, Proposition 1.1], we have

$$
\Lambda_{k}(A)=\left\{\lambda \in \mathbb{C}: X^{*} A X=\lambda I_{k}, \text { for some } X \in \mathcal{X}_{n, k}\right\},
$$

where $\mathcal{X}_{n, k}=\left\{X \in M_{n \times k}: X^{*} X=I_{k}\right\}$, i.e., the set of all $n \times k$ isometry matrices. The sets $\Lambda_{k}(A)$, where $k \in\{1, \ldots, n\}$, are generally called higher rank numerical ranges of $A$. Apparently, for $k=1, \Lambda_{k}(A)$ reduces to the classical numerical range of $A$, namely,

$$
\Lambda_{1}(A)=W(A):=\left\{x^{*} A x: x \in \mathbb{C}^{n}, x^{*} x=1\right\},
$$

which has been studied extensively for many decades, and several interesting results about some generalizations of this notion to matrix polynomials, which play an important role in the study of overdamped vibration systems with a finite number of degrees of freedom, and they are also related to the stability theory, have been obtained, e.g., see [1] and [13], and references cited there. Also, the concept of numerical range is useful in studying and understanding of matrices and operators, and has many applications in numerical analysis, differential equations, systems theory, etc; e.g., see [9, 10] and their references. It is readily verified that

$$
W(A)=\Lambda_{1}(A) \supseteq \Lambda_{2}(A) \supseteq \cdots \supseteq \Lambda_{n}(A) .
$$

The numerical range $W(A)=\Lambda_{1}(A)$ is a nonempty, compact and convex subset of $\mathbb{C}$, and it contains the spectrum of $A$. The higher rank numerical ranges can be empty, and also there are conditions for which $\Lambda_{k}(A)$ is non-empty; e.g., see [12]. But the compactness and convexity still hold in general by the following, see [2], fact:

$$
\Lambda_{k}(A)=\bigcap_{X \in \mathcal{X}_{n, n-k+1}} W\left(X^{*} A X\right) .
$$

Stampfli and Williams in [14, Theorem 4], and later Bonsall and Duncan in [3, Lemma 6.22.1], observed that the numerical range of $A \in M_{n}$ can be rewritten as:

$$
W(A)=\left\{\mu \in \mathbb{C}:\left\|A-\lambda I_{n}\right\|_{2} \geq|\mu-\lambda|, \quad \forall \lambda \in \mathbb{C}\right\},
$$

where $\|.\|_{2}$ denotes the spectral matrix norm (i.e., the matrix norm subordinate to the Euclidean vector norm), and $I_{n}$ is the $n \times n$ identity matrix. By this idea, Chorianopoulos, Karanasios and Psarrakos [6] recently introduced a definition of the numerical range for rectangular complex matrices. For any $A, B \in M_{n \times m}$ with $B \neq 0$, and any vector norm $\|$.$\| on M_{n \times m}$, they defined the numerical range of $A$ with respect to $B$ as the compact and convex set:

$$
W_{\|\cdot\|}(A ; B)=\{\mu \in \mathbb{C}:\|A-\lambda B\| \geq|\mu-\lambda|, \quad \forall \lambda \in \mathbb{C}\} .
$$

It is clear that $W_{\|\cdot\|_{2}}\left(A ; I_{n}\right)=W(A)=\Lambda_{1}(A)$, where $A \in M_{n}$. Hence, $W_{\|\cdot\|}(. ;$. is a direct generalization of the classical numerical range. It is known that $W_{\|\cdot\|}(A ; B) \neq \emptyset$ if and only if $\|B\| \geq 1$. So, to avoid trivial consideration, we assume that $\|B\| \geq 1$. Next, we list some properties of $W_{\|\cdot\|}(. ;$.$) , which will be$ useful in our discussion. One may see [6] and [7] for more details. Recall that, in a complex normed space $(X,\|\cdot\|)$, for any $\epsilon \in[0,1)$, two vectors $\phi$ and $\psi$ are said to be Birkhoff-James $\epsilon$-orthogonal, denoted by $\varphi \perp_{B J}^{\epsilon} \psi$, if $\|\varphi+\lambda \psi\| \geq \sqrt{1-\epsilon^{2}}\|\varphi\|$ for all $\lambda \in \mathbb{C}$. For the case $\epsilon=0$, we write $\varphi \perp_{B J} \psi$ instead of $\varphi \perp_{B J}^{0} \psi$. 
Proposition 1.1. Let $A, B \in M_{n \times m}$, and $\|$.$\| be a vector norm on M_{n \times m}$. Then the following assertions are true:

(i) If $\|B\|>1$, then $\left\{\mu \in \mathbb{C}: B \perp_{B J}(A-\mu B)\right\} \subseteq W_{\|\cdot\|}(A ; B)$. The equality holds if $\|B\|=1$;

(ii) If the norm $\|$.$\| is unitarily invariant, then W_{\|.\|}(U A V ; U B V)=W_{\|.\|}(A ; B)$, where $U \in \mathcal{U}_{n}$ and $V \in \mathcal{U}_{m}$;

(iii) $W_{\|.\|}(a A+b B ; B)=a W_{\|.\|}(A ; B)+b$, where $a, b \in \mathbb{C}$;

(iv) $\left\{\mu^{-1} \in \mathbb{C}: \mu \in W_{\|.\|}(A ; B),|\mu| \geq 1\right\} \subseteq W_{\|.\|}(B ; A)$;

(v) $\operatorname{Int}\left(W_{\|\cdot\|}(A ; B)\right) \subseteq\{\mu \in \mathbb{C}:\|A-\lambda B\|>|\mu-\lambda|, \forall \lambda \in \mathbb{C}\}$, where $\operatorname{Int}($. denotes the interior points;

(vi) For any nonzero $b \in \mathbb{C}$,

$$
\left\{\begin{array}{l}
\text { if }|b|=1, \text { then } W_{\|\cdot\|}(A ; b B)=b^{-1} W_{\|\cdot\|}(A ; B) ; \\
\text { if }|b|<1, \text { then } W_{\|\cdot\|}(A ; b B) \subseteq b^{-1} W_{\|\cdot\|}(A ; B) ; \\
\text { if }|b|>1, \text { then } W_{\|\cdot\|}(A ; b B) \supseteq b^{-1} W_{\|\cdot\|}(A ; B) ;
\end{array}\right.
$$

(vii) If $A=b B$ for some $b \in \mathbb{C}$, then $W_{\|\cdot\|}(A ; B)=\{b\}$.

In section 2 of this paper, we introduce the notion of rank $-k$ numerical range of rectangular complex matrices as a generalization of $W_{\|.\|}(. ;$.$) . We investigate$ some general properties of the notion, and we also introduce and study the notion of $\epsilon$-higher rank numerical ranges of rectangular matrices. In section 3 , we introduce and study the notion of $k$-spectrum of rectangular matrices. In particular, we state the relationship between this notion and rank $-k$ numerical range.

\section{RANK $-k$ NUMERICAL RANGE OF RECTANGULAR MATRICES}

It is natural to use a formula analogous to (1.2) to propose a definition of the higher rank numerical range of rectangular matrices. For this mind, for positive integers $m, n$ and $1 \leq k \leq \min \{n, m\}$, we introduce the following set:

$$
\begin{cases}\mathcal{X}=\left\{\left(X, Y:=\left[\begin{array}{c|c}
X & 0 \\
\hline 0 & U
\end{array}\right]\right): X \in \mathcal{X}_{n, n-k+1}, U \in \mathcal{U}_{m-n}\right\} \quad \text { if } m \geq n, \\
\mathcal{X}=\left\{\left(X:=\left[\begin{array}{c|c}
Y & 0 \\
\hline 0 & U
\end{array}\right], Y\right): Y \in \mathcal{X}_{m, m-k+1}, U \in \mathcal{U}_{n-m}\right\} & \text { if } n \geq m .\end{cases}
$$

Definition 2.1. Let $A, B \in M_{n \times m}, 1 \leq k \leq \min \{n, m\}$ be a positive integer, and $\mathcal{X}$ be the set as in (2.1). Moreover, let $\|\cdot\|$ be a vector norm on $M_{(n-k+1) \times(m-k+1)}$. The rank $-k$ numerical range of $A$ with respect to $B$ is defined and denoted by

$$
\Lambda_{k,\|\cdot\|}(A ; B)=\left\{\mu \in \mathbb{C}:\left\|X^{*}(A-\lambda B) Y\right\| \geq|\mu-\lambda|, \forall \lambda \in \mathbb{C}, \forall(X, Y) \in \mathcal{X}\right\} .
$$

In the following theorem, we give a formula, analogous to (1.1), for $\Lambda_{k,\|\cdot\|}(.,$.$) .$

Theorem 2.2. Let $A, B \in M_{n \times m}$ and $1 \leq k \leq \min \{n, m\}$ be a positive integer. Moreover, let $\|\cdot\|$ be a vector norm on $M_{(n-k+1) \times(m-k+1)}$. Then

$$
\Lambda_{k,\|\cdot\|}(A ; B)=\bigcap_{(X, Y) \in \mathcal{X}} W_{\|\cdot\|}\left(X^{*} A Y ; X^{*} B Y\right)
$$


where $\mathcal{X}$ is the set as in (2.1). Consequently, $\Lambda_{k,\|\cdot\|}(A ; B)$ is a compact and convex set in $\mathbb{C}$. For the case $k=1$, if the vector norm $\|\cdot\|$ is unitarily invariant, then

$$
\Lambda_{1}(A ; B)=W_{\|\cdot\|}(A ; B) .
$$

Proof. Using Definition 2.1 and relation (1.2), the first equality is easy to verify. Since, for any $(X, Y) \in \mathcal{X}, W_{\|\cdot\|}\left(X^{*} A Y ; X^{*} B Y\right)$ is a compact and convex set in $\mathbb{C}, \Lambda_{k,\|\cdot\|}(A ; B)$ is a compact and convex set. If $k=1$ and the vector norm $\|\cdot\|$ is unitarily invariant on $M_{n \times m}$, then by Proposition $1.1(i i)$, the second equality can be easily verify by the first result.

Corollary 2.3. Let $A, B \in M_{n}$ and $1 \leq k \leq n$ be a positive integer. Moreover, let $\|\cdot\|$ be a vector norm on $M_{n-k+1}$. Then

$$
\Lambda_{k,\|\cdot\|}(A ; B)=\bigcap_{X \in \mathcal{X}_{n, n-k+1}} W_{\|\cdot\|}\left(X^{*} A X ; X^{*} B X\right)
$$

Consequently, for the case $\|\cdot\|=\|\cdot\|_{2}$ and $B=I_{n}$,

$$
\Lambda_{k,\|\cdot\|}\left(A ; I_{n}\right)=\Lambda_{k}(A) \text {. }
$$

Remark 2.4. By [6, Corollary 4], for every $A, B \in M_{n \times m}$, we have $W_{\|\cdot\|}(A ; B) \neq$ $\emptyset$ if and only if $\|B\| \geq 1$. So, in view of Theorem 2.2, to avoid of trivial cases, in this paper, we assume that $\left\|X^{*} B Y\right\| \geq 1$ for all $(X, Y) \in \mathcal{X}$. By [12, Theorem 3], if $k \geq \frac{n}{3}+1$, then there exists $A \in M_{n}$ such that $\Lambda_{k}(A)=\emptyset$. Hence, by Corollary 2.3, $\Lambda_{k,\|\cdot\|_{2}}\left(A ; I_{n}\right)=\Lambda_{k}(A)=\emptyset$. Also, by Proposition 1.1(vii) and Theorem 2.2, $\Lambda_{k,\|\cdot\|}(A ; A)=\{1\}$ for any $A \in M_{n \times m}$, and hence, $\Lambda_{k,\|\cdot\|}(. ;$.$) can be empty or a$ non-empty set.

Remark 2.5. By Corollary 2.3, the notion of rank- $k$ numerical range of rectangular matrices can be considered as a generalization of the rank $-k$ numerical range of square matrices.

In the following theorem, we show that the rank- $k$ numerical range of rectangular matrices is invariant under some unitary matrices.

Theorem 2.6. Let $A, B \in M_{n \times m}$ and $1 \leq k \leq \min \{n, m\}$ be a positive integer. Moreover, let $\|\cdot\|$ be a vector norm on $M_{(n-k+1) \times(m-k+1)}$. Then

$$
\Lambda_{k,\|\cdot\|}(U A V ; U B V)=\Lambda_{k,\|\cdot\|}(A ; B)
$$

where for the case $m \geq n, U \in \mathcal{U}_{n}$ and $V=\left(\begin{array}{c|c}U^{*} & 0 \\ \hline 0 & *\end{array}\right) \in \mathcal{U}_{m}$, and for the other case, i.e., $n \geq m, V \in \mathcal{U}_{m}$ and $U=\left(\begin{array}{c|c}V^{*} & 0 \\ \hline 0 & *\end{array}\right) \in \mathcal{U}_{n}$.

Proof. Without loss of generality, we assume that $m \geq n$. To prove $\subseteq$, let $\mathcal{X}$ be the set as in (2.1), and let $(X, Y) \in \mathcal{X}$ be given. So, $X \in \mathcal{X}_{n, n-k+1}$ and $Y=\left(\begin{array}{c|c}X & 0 \\ \hline 0 & W\end{array}\right)$ for some $W \in \mathcal{U}_{m-n}$. By setting $X^{\prime}:=U X$ and $Y^{\prime}:=V^{*} Y$, we have $X^{\prime} \in \mathcal{X}_{n, n-k+1}$ and $Y^{\prime}=\left(\begin{array}{c|c}U X & 0 \\ \hline 0 & * \in \mathcal{U}_{m-n}\end{array}\right)=\left(\begin{array}{c|c}X^{\prime} & 0 \\ \hline 0 & * \in \mathcal{U}_{m-n}\end{array}\right)$. So, 
$\left(X^{\prime}, Y^{\prime}\right) \in \mathcal{X}$, and hence, by Theorem 2.2, we have

$$
\begin{aligned}
\Lambda_{k,\|\cdot\|}(U A V ; U B V) & \subseteq W_{\|\cdot\|}\left(\left(X^{\prime}\right)^{*}(U A V) Y^{\prime} ;\left(X^{\prime}\right)^{*}(U B V) Y^{\prime}\right) \\
& =W_{\|\cdot\|}\left(X^{*} A Y ; X^{*} B Y\right) .
\end{aligned}
$$

Since $(X, Y) \in \mathcal{X}$ is arbitrary, by Theorem $2.2, \Lambda_{k,\|\cdot\|}(U A V ; U B V) \subseteq \Lambda_{k,\|\cdot\|}(A ; B)$. By a similar way, $\supseteq$ can be easily verified. So, the proof is complete.

Let $n, m \in \mathbb{N}$ and $1 \leq k_{2} \leq k_{1} \leq \min \{n, m\}$ be two positive integers. Moreover, let $\|\cdot\|$ be a vector norm on $M_{\left(n-k_{2}+1\right) \times\left(m-k_{2}+1\right)}$. Define $\||\cdot|\|$ on $M_{\left(n-k_{1}+1\right) \times\left(m-k_{1}+1\right)}$ by

$$
\|Z\|\|=\|\left(\begin{array}{c|c}
Z & 0 \\
\hline 0 & 0_{k_{1}-k_{2}}
\end{array}\right) \|,
$$

where $Z \in M_{\left(n-k_{1}+1\right) \times\left(m-k_{1}+1\right)}$, and $0_{k_{1}-k_{2}} \in M_{k_{1}-k_{2}}$ is the zero matrix. Now, in the following theorem, we want to study the relationship between higher rank numerical ranges.

Theorem 2.7. Let $A, B \in M_{n \times m}$ and $1 \leq k_{2} \leq k_{1} \leq \min \{n, m\}$ be two positive integers. Moreover, let $\|\cdot\|$ be a unitarily invariant norm on $M_{\left(n-k_{2}+1\right) \times\left(m-k_{2}+1\right)}$ and $\||\cdot|\|$ be the vector norm on $M_{\left(n-k_{1}+1\right) \times\left(m-k_{1}+1\right)}$ as in (2.2). Then

$$
\Lambda_{k_{1},\||\cdot|\|}(A ; B) \subseteq \Lambda_{k_{2},\|\cdot\|}(A ; B) .
$$

Proof. Let $\mu \in \Lambda_{k_{1},\|\cdot \cdot\|}(A ; B)$ be given. Without loss of generality, we assume that $n \geq m$, and

$$
A=\left(\frac{A_{1}}{A_{2}}\right), B=\left(\frac{B_{1}}{B_{2}}\right) \in M_{n \times m},
$$

where $A_{1}, B_{1} \in M_{m}$ and $A_{2}, B_{2} \in M_{(n-m) \times m}$. In view of Definition 2.1, it is enough to show that for every $Y \in \mathcal{X}_{m, m-k_{2}+1}, U \in \mathcal{U}_{n-m}$ and $\lambda \in \mathbb{C}$,

$$
\left\|X^{*}(A-\lambda B) Y\right\| \geq|\mu-\lambda|
$$

where $X=\left(\begin{array}{c|c}Y & 0 \\ \hline 0 & U\end{array}\right)$.

For this mind, let $Y \in \mathcal{X}_{m, m-k_{2}+1}, U \in \mathcal{U}_{n-m}$ and $\lambda \in \mathbb{C}$ be given. By setting $Y=\left(Y_{1} \mid Y_{2}\right)$, where $Y_{1} \in M_{m \times\left(m-k_{1}+1\right)}$ and $Y_{2} \in M_{m \times\left(k_{1}-k_{2}\right)}$, we have $Y_{1} \in \mathcal{X}_{m, m-k_{1}+1}$. Moreover,

$$
\begin{aligned}
\left\|X^{*}(A-\lambda B) Y\right\| & =\left\|\left(\begin{array}{c|c}
Y_{1}^{*}\left(A_{1}-\lambda B_{1}\right) Y_{1} & Y_{1}^{*}\left(A_{1}-\lambda B_{1}\right) Y_{2} \\
Y_{2}^{*}\left(A_{1}-\lambda B_{1}\right) Y_{1} & Y_{2}^{*}\left(A_{1}-\lambda B_{1}\right) Y_{2} \\
\hline U^{*}\left(A_{2}-\lambda B_{2}\right) Y_{1} & U^{*}\left(A_{2}-\lambda B_{2}\right) Y_{2}
\end{array}\right)\right\| \\
& =\left\|\left(\begin{array}{c|c}
Y_{1}^{*}\left(A_{1}-\lambda B_{1}\right) Y_{1} & Y_{1}^{*}\left(A_{1}-\lambda B_{1}\right) Y_{2} \\
U^{*}\left(A_{2}-\lambda B_{2}\right) Y_{1} & U^{*}\left(A_{2}-\lambda B_{2}\right) Y_{2} \\
\hline Y_{2}^{*}\left(A_{1}-\lambda B_{1}\right) Y_{1} & Y_{2}^{*}\left(A_{1}-\lambda B_{1}\right) Y_{2}
\end{array}\right)\right\|,
\end{aligned}
$$


in which, the second equality follows from this fact that $\|\cdot\|$ is unitarily invariant. Hence, by [15, Corollary 3.8], we have

$$
\begin{aligned}
\left\|X^{*}(A-\lambda B) Y\right\| & \geq\left\|\left(\begin{array}{c|c}
Y_{1}^{*}\left(A_{1}-\lambda B_{1}\right) Y_{1} & 0 \\
U^{*}\left(A_{2}-\lambda B_{2}\right) Y_{1} & 0 \\
\hline 0 & 0
\end{array}\right)\right\| \\
& =\left\|\left(\begin{array}{c|c}
X_{1}^{*}(A-\lambda B) Y_{1} & 0 \\
\hline 0 & 0
\end{array}\right)\right\| \\
& =\left\|X_{1}^{*}(A-\lambda B) Y_{1}\right\|,
\end{aligned}
$$

where $X_{1}=\left(\begin{array}{c|c}Y_{1} & 0 \\ \hline 0 & U\end{array}\right) \in \mathcal{X}_{n, n-k_{1}+1}$. Since $\mu \in \Lambda_{k_{1},\||\cdot|\|}(A ; B), \quad \| X_{1}^{*}(A-$ $\lambda B) Y_{1}|||\geq| \mu-\lambda \mid$, and hence the above inequality shows that

$$
\left\|X^{*}(A-\lambda B) Y\right\| \geq|\mu-\lambda| .
$$

So, the proof is complete.

Proposition 2.8. Let $A, B \in M_{n \times m}$ and $1 \leq k \leq \min \{n, m\}$ be a positive integer. Moreover, let $\|\cdot\|$ be a vector norm on $M_{(n-k+1) \times(m-k+1)}$ and $\mathcal{X}$ be the set as in (2.1). Then the following assertions are true:

(i) If $\left\|X^{*} B Y\right\|=1$ for all $(X, Y) \in \mathcal{X}$, then

$$
\Lambda_{k,\|\cdot\|}(A ; B)=\bigcap_{(X, Y) \in \mathcal{X}}\left\{\mu \in \mathbb{C}: X^{*} B Y \perp_{B J} X^{*}(A-\mu B) Y\right\}
$$

(ii) If $\left\|X^{*} B Y\right\|>1$ for all $(X, Y) \in \mathcal{X}$, then

$$
\Lambda_{k,\|\cdot\|}(A ; B) \supseteq \bigcap_{(X, Y) \in \mathcal{X}}\left\{\mu \in \mathbb{C}: X^{*} B Y \perp_{B J} X^{*}(A-\mu B) Y\right\} .
$$

Proof. Let $(X, Y) \in \mathcal{X}$ be arbitrary. By Proposition 1.1(i), if $\left\|X^{*} B Y\right\|=1$, then $\left.\left\{\mu \in \mathbb{C}: X^{*} B Y \perp_{B J} X^{*}(A-\mu B) Y\right\}=W_{\|\cdot\|}\left(X^{*} A Y ; X^{*} B Y\right)\right)$; and if $\left\|X^{*} B Y\right\|>1$, then $\left.\left\{\mu \in \mathbb{C}: X^{*} B Y \perp_{B J} X^{*}(A-\mu B) Y\right\} \subseteq W_{\|\cdot\|}\left(X^{*} A Y ; X^{*} B Y\right)\right)$. Now, the results follow from Theorem 2.2 .

The following proposition follows from Proposition 1.1(vi) and Theorem 2.2.

Proposition 2.9. Let $A, B \in M_{n \times m}, \quad 0 \neq b \in \mathbb{C}$, and $1 \leq k \leq \min \{n, m\}$ be $a$ positive integer. Moreover, let $\|\cdot\|$ be a vector norm on $M_{(n-k+1) \times(m-k+1)}$. Then the following assertions are true:

(i) If $|b|=1$, then $\Lambda_{k,\|\cdot\|}(A ; b B)=b^{-1} \Lambda_{k,\|\cdot\|}(A ; B)$;

(ii) If $|b|<1$, then $\Lambda_{k,\|\cdot\|}(A ; b B) \subseteq b^{-1} \Lambda_{k,\|\cdot\|}(A ; B)$;

(iii) If $|b|>1$, then $\Lambda_{k,\|\cdot\|}(A ; b B) \supseteq b^{-1} \Lambda_{k,\|\cdot\|}(A ; B)$.

The following proposition follows from Proposition 1.1(iii) and Theorem 2.2.

Proposition 2.10. Let $A, B \in M_{n \times m}$ and $1 \leq k \leq \min \{n, m\}$ be a positive integer. Moreover, let $\|\cdot\|$ be a vector norm on $M_{(n-k+1) \times(m-k+1)}$, and $a, b \in \mathbb{C}$. Then $\Lambda_{k,\|\cdot\|}(a A+b B ; B)=a \Lambda_{k,\|\cdot\|}(A ; B)+b$. 
Proposition 2.11. Let $A, B \in M_{n \times m}$ and $1 \leq k \leq \min \{n, m\}$ be a positive integer. Moreover, let $\|\cdot\|$ be a vector norm on $M_{(n-k+1) \times(m-k+1)}$. Then

$$
\operatorname{Int}\left(\Lambda_{k,\|\cdot\|}(A ; B)\right) \subseteq \bigcap_{(X, Y) \in \mathcal{X}}\left\{\mu \in \mathbb{C}:\left\|X^{*}(A-\lambda B) Y\right\|>|\mu-\lambda|, \quad \forall \lambda \in \mathbb{C}\right\},
$$

where $\operatorname{Int}(S)$ denotes the set of all interior points of $S \subseteq \mathbb{C}$.

Proof. Since

$$
\operatorname{Int}\left(\bigcap_{(X, Y) \in \mathcal{X}} W_{\|\cdot\|}\left(X^{*} A Y ; X^{*} B Y\right)\right) \subseteq \bigcap_{(X, Y) \in \mathcal{X}} \operatorname{Int}\left(W_{\|\cdot\|}\left(X^{*} A Y ; X^{*} B Y\right)\right),
$$

using Theorem 2.2 and Proposition 1.1(v), the result holds.

In the following proposition, we state the relationship between the $\Lambda_{k,\|\cdot\|}(A ; B)$ and $\Lambda_{k,\|\cdot\|}(B ; A)$.

Proposition 2.12. Let $A, B \in M_{n \times m}$ and $1 \leq k \leq \min \{n, m\}$ be a positive integer. Moreover, let $\|\cdot\|$ be a vector norm on $M_{(n-k+1) \times(m-k+1)}$. Then

$$
\left\{\mu^{-1}: \mu \in \Lambda_{k,\|\cdot\|}(A ; B),|\mu| \geq 1\right\} \subseteq \Lambda_{k,\|\cdot\|}(B ; A) .
$$

Proof. By Proposition 1.1(iv) and Theorem 2.2, we have

$$
\begin{aligned}
\bigcap_{(X, Y) \in \mathcal{X}}\left\{\mu^{-1}: \mu \in W_{\|\cdot\|}\left(X^{*} A Y ; X^{*} B Y\right),|\mu| \geq 1\right\} & \subseteq \bigcap_{(X, Y) \in \mathcal{X}} W_{\|\cdot\|}\left(X^{*} B Y ; X^{*} A Y\right) \\
& =\Lambda_{k,\|\cdot\|}(B ; A) .
\end{aligned}
$$

Since

$$
\begin{gathered}
\left\{\mu^{-1}: \mu \in \Lambda_{k,\|\cdot\|}(A ; B),|\mu| \geq 1\right\} \subseteq \bigcap_{(X, Y) \in \mathcal{X}}\left\{\mu^{-1} \in \mathbb{C} \quad: \mu \in W_{\|\cdot\|}\left(X^{*} A Y ; X^{*} B Y\right)\right. \\
, \quad|\mu| \geq 1\},
\end{gathered}
$$

by the above inclusion, the result holds.

Let $A, B \in M_{n \times m}$ and $0 \leq \epsilon<1$. Moreover, let $\|\cdot\|$ be a vector norm on $M_{n \times m}$. The Birkhoff-James $\epsilon$-orthogonality set of $A$ with respect to $B$ is defined and denoted, [7, Definition 1], by

$$
W_{\|\cdot\|}^{\epsilon}(A ; B)=\left\{\mu \in \mathbb{C}:\|A-\lambda B\| \geq \sqrt{1-\epsilon^{2}}\|B\||\mu-\lambda|, \forall \lambda \in \mathbb{C}\right\} .
$$

It is clear that:

$$
W_{\|\cdot\|}^{\epsilon}(A ; B)=\left\{\mu \in \mathbb{C}: B \perp_{B J}^{\epsilon}(A-\mu B)\right\}
$$

By this idea, at the end of this section, we introduce and study the notion of rank $-k, \epsilon$ numerical range of rectangular matrices.

Definition 2.13. Let $A, B \in M_{n \times m}, 1 \leq k \leq \min \{n, m\}$ be a positive integer, and $\mathcal{X}$ be the set as in (2.1). Moreover, let $\|\cdot\|$ be a vector norm on $M_{(n-k+1) \times(m-k+1)}$ and $0 \leq \epsilon<1$. The rank-k, $\epsilon$ numerical range of $A$ with respect to $B$ is defined and denoted by

$$
\begin{aligned}
\Lambda_{k,\|\cdot\|}^{\epsilon}(A ; B)=\{\mu \in \mathbb{C}: & \left\|X^{*}(A-\lambda B) Y\right\| \geq \sqrt{1-\epsilon^{2}}\left\|X^{*} B Y\right\| \| \mu-\lambda \mid, \forall \lambda \in \mathbb{C}, \\
& \forall(X, Y) \in \mathcal{X}\} .
\end{aligned}
$$


Theorem 2.14. Let $A, B \in M_{n \times m}, 1 \leq k \leq \min \{n, m\}$ be a positive integer, and $\mathcal{X}$ be the set as in (2.1). Moreover, let $\|\cdot\|$ be a vector norm on $M_{(n-k+1) \times(m-k+1)}$ and $0 \leq \epsilon<1$. Then

$$
\Lambda_{k,\|\cdot\|}^{\epsilon}(A ; B)=\bigcap_{(X, Y) \in \mathcal{X}} W_{\|\cdot\|}^{\epsilon}\left(X^{*} A Y ; X^{*} B Y\right) .
$$

Consequently, $\Lambda_{k,\|\cdot\|}^{\epsilon}(A ; B)$ is a compact and convex set in $\mathbb{C}$.

Proof. Using Definition 2.13 and Relation (2.3), the equality is easy to verify. Since $W_{\|\cdot\|}^{\epsilon}\left(X^{*} A Y ; X^{*} B Y\right)$ is a compact and convex set in $\mathbb{C}$ for all $(X, Y) \in \mathcal{X}$, the convexity and compactness of $\Lambda_{k,\|\cdot\|}^{\epsilon}(A ; B)$ follows.

Using Theorem 2.14 and relation (2.4), we have the following proposition.

Proposition 2.15. Let $A, B \in M_{n \times m}$ and $1 \leq k \leq \min \{n, m\}$ be a positive integer. Moreover, let $\|\cdot\|$ be a vector norm on $M_{(n-k+1) \times(m-k+1)}$ and $0 \leq \epsilon<1$. Then

$$
\Lambda_{k,\|\cdot\|}^{\epsilon}(A ; B)=\bigcap_{(X, Y) \in \mathcal{X}}\left\{\mu \in \mathbb{C}: X^{*} B Y \perp_{B J}^{\epsilon} X^{*}(A-\mu B) Y\right\} .
$$

\section{3. $k$-Spectrum of RECTANGUlar MATRICES}

Let $A, B \in M_{n \times m}$, where $n \geq m$. A scalar $\mu_{0} \in \mathbb{C}$ is said to be an eigenvalue of $A$ with respect to $B$ if the linear system $\left(A-\mu_{0} B\right) x=0$ has a nonzero solution $x_{0} \in \mathbb{C}^{m}$. The vector $x_{0}$ is called an eigenvector of $A$ with respect to $B$ corresponding to $\mu_{0}$, and the set of all eigenvalues of $A$ with respect to $B$ is denoted by $\sigma(A ; B)$. One may see [6] for more details. It is clear that

$$
\sigma(A ; B)=\{\mu \in \mathbb{C}: \operatorname{dim} \operatorname{ker}(A-\mu B) \geq 1\} .
$$

By this idea, we state the following definition. We use the notation $[x]$ for the greatest integer less than or equal to $x \in \mathbb{R}$.

Definition 3.1. Let $A, B \in M_{n \times m}$, where $n \geq m$, and let $1 \leq k \leq\left[\frac{m+1}{2}\right]$ be a positive integer, and $\mathcal{X}$ be the set as in (2.1). The $k$ - spectrum of $A$ with respect to $B$ is defined and denoted by

$$
\sigma_{k}(A ; B)=\left\{\mu \in \mathbb{C}: \operatorname{dim} \operatorname{ker}\left(X^{*}(A-\mu B) Y\right) \geq k, \quad \forall(X, Y) \in \mathcal{X}\right\} .
$$

Remark 3.2. In Definition 3.1, if $A, B \in M_{n}$ and $1 \leq k \leq\left[\frac{n+1}{2}\right]$ is a positive integer, then

$$
\sigma_{k}(A ; B)=\left\{\mu \in \mathbb{C}: \operatorname{dim} \operatorname{ker}\left(X^{*}(A-\mu B) X\right) \geq k, \quad \forall X \in \mathcal{X}_{n, n-k+1}\right\} .
$$

So, if $B=I_{n}$, then $\sigma_{1}\left(A ; I_{n}\right)=\sigma(A)$, and hence, the notion of $k$-spectrum can be considered as a generalization of the spectrum.

Theorem 3.3. Let $A, B \in M_{n \times m}$, where $n \geq m$, and let $1 \leq k \leq\left[\frac{m+1}{2}\right]$ be a positive integer. Then the following assertions are true: 
(i) $\sigma(A ; B)=\sigma_{1}(A ; B) \supseteq \sigma_{2}(A ; B) \supseteq \cdots \supseteq \sigma_{\left[\frac{m+1}{2}\right]}(A ; B)$;

(ii) $\left\{\mu^{-1} \in \mathbb{C}: \mu \in \sigma_{k}(A ; B)\right.$ and $\left.\mu \neq 0\right\}=\sigma_{k}(B ; A) \backslash\{0\}$;

(iii) $\sigma_{k}\left(A^{*} ; B^{*}\right)=\overline{\sigma_{k}(A ; B)}:=\left\{\bar{\mu} \in \mathbb{C}: \mu \in \sigma_{k}(A ; B)\right\}$, where $n=m$.

Proof. $(i)$; The equality $\sigma_{1}(A ; B)=\sigma(A ; B)$ follows from this fact that for any unitary matrices $X \in M_{n}$ and $Y \in M_{m}$, dim $\operatorname{ker}(A-\mu B)=\operatorname{dim} \operatorname{ker}\left(X^{*}(A-\right.$ $\mu B) Y)$.

Now, let $1 \leq k_{1} \leq k_{2} \leq\left[\frac{m+1}{2}\right]$ be two positive integers. We will show that $\sigma_{k_{2}}(A ; B) \subseteq \sigma_{k_{1}}(A ; B)$. For this mind, let $\mu \in \sigma_{k_{2}}(A ; B), Y \in \mathcal{X}_{m, m-k_{1}+1}$ and $U \in$ $\mathcal{U}_{n-m}$ be given. By setting $Y=\left(Y_{1} \mid Y_{2}\right), A=\left(\frac{A_{1}}{A_{2}}\right), B=\left(\frac{B_{1}}{B_{2}}\right) \in M_{n \times m}$, where $Y_{1} \in M_{m \times\left(m-k_{2}+1\right)}, Y_{2} \in M_{m \times\left(k_{2}-k_{1}\right)}$, and $A_{1}, B_{1} \in M_{m}$ and $A_{2}, B_{2} \in$ $M_{(n-m) \times m}$, we have $Y_{1} \in \mathcal{X}_{m, m-k_{2}+1}$, and

$\operatorname{rank}\left(X^{*}(A-\mu B) Y\right)=\operatorname{rank}\left(\begin{array}{c|c}Y_{1}^{*}\left(A_{1}-\mu B_{1}\right) Y_{1} & Y_{1}^{*}\left(A_{1}-\mu B_{1}\right) Y_{2} \\ U^{*}\left(A_{2}-\mu B_{2}\right) Y_{1} & U^{*}\left(A_{2}-\mu B_{2}\right) Y_{2} \\ \hline Y_{2}^{*}\left(A_{1}-\mu B_{1}\right) Y_{1} & Y_{2}^{*}\left(A_{1}-\mu B_{1}\right) Y_{2}\end{array}\right)$,

where $X=\left(\begin{array}{c|c}Y & 0 \\ \hline 0 & U\end{array}\right) \in \mathcal{X}_{n, n-k_{1}+1}$. So,

$$
\operatorname{rank}\left(X^{*}(A-\mu B) Y\right)=\operatorname{rank}\left(\begin{array}{c|c}
X_{1}^{*}(A-\mu B) Y_{1} & Y_{1}^{*}\left(A_{1}-\mu B_{1}\right) Y_{2} \\
& U^{*}\left(A_{2}-\mu B_{2}\right) Y_{2} \\
\hline Y_{2}^{*}\left(A_{1}-\mu B_{1}\right) Y_{1} & Y_{2}^{*}\left(A_{1}-\mu B_{1}\right) Y_{2}
\end{array}\right),
$$

where $X_{1}=\left(\begin{array}{c|c}Y_{1} & 0 \\ \hline 0 & U\end{array}\right) \in \mathcal{X}_{n, n-k_{2}+1}$. Since $\mu \in \sigma_{k_{2}}(A ; B), \operatorname{dim} \operatorname{ker}\left(X_{1}^{*}(A-\right.$ $\left.\mu B) Y_{1}\right) \geq k_{2}$, and hence, $\operatorname{rank}\left(X_{1}^{*}(A-\mu B) Y_{1}\right) \leq m-2 k_{2}+1$. So, the inequality $k_{2} \geq k_{1}$ implies that

$$
\begin{aligned}
\operatorname{rank}\left(X^{*}(A-\mu B) Y\right) & \leq \operatorname{rank}\left(X_{1}^{*}(A-\mu B) Y_{1}\right)+\left(k_{2}-k_{1}\right) \\
& \leq m-k_{1}-k_{2}+1 \\
& \leq m-2 k_{1}+1 .
\end{aligned}
$$

Therefore, $\operatorname{dim} \operatorname{ker}\left(X^{*}(A-\mu B) Y\right) \geq k_{1}$, and hence, $\mu \in \sigma_{k_{1}}(A ; B)$.

Using Definition 3.1, the results in $(i i)$ and (iii) can be easily verified.

In the final theorem, we state the relationship between $\sigma_{k}(A ; B)$ and $\Lambda_{k,\|\cdot\|}(A ; B)$.

Theorem 3.4. Let $A, B \in M_{n \times m}$, where $n \geq m$, and let $1 \leq k \leq\left[\frac{m+1}{2}\right]$ be a positive integer. Moreover, let $\|\cdot\|$ be an induced vector norm on $M_{(n-k+1) \times(m-k+1)}$ such that $\left\|X^{*} B Y z\right\| \geq 1$ for all $(X, Y) \in \mathcal{X}$ and $z \in \mathbb{C}^{m-k+1} \backslash\{0\}$, where $\mathcal{X}$ is the set as in (2.1). Then

$$
\sigma_{k}(A ; B) \subseteq \Lambda_{k,\|\cdot\|}(A ; B)
$$


Proof. Let $\mu \in \sigma_{k}(A ; B),(X, Y) \in \mathcal{X}$ and $\lambda \in \mathbb{C}$ be given. By Definition 3.1, there exists a unit vector $z \in \mathbb{C}^{m-k+1} \backslash\{0\}$ such that $X^{*}(A-\mu B) Y z=0$. So $X^{*}(A-\lambda B) Y z=(\mu-\lambda) X^{*} B Y z$, and hence,

$$
\left\|X^{*}(A-\lambda B) Y\right\| \geq\left\|X^{*}(A-\lambda B) Y z\right\|=|\mu-\lambda|\left\|X^{*} B Y z\right\| \geq|\mu-\lambda| .
$$

So, $\mu \in \Lambda_{k,\|\cdot\|}(A ; B)$ and hence, the proof is complete.

Acknowledgement. The authors wish to express their gratitude to anonymous referees for helpful comments and useful suggestions. The second author also thanks the Mahani Mathematical Research Center of Shahid Bahonar University of Kerman, Kerman, Iran.

\section{REFERENCES}

1. Gh. Aghamollaei and A. Salemi, Polynomial numerical hulls of matrix polynomials, II, Linear Multilinear Algebra 59 (2011), 291-302.

2. A. Aretaki and J. Maroulas, The rank-k numerical radii, Ann. Funct. Anal. 3 (2012), 100-108.

3. F.F. Bonsall and J. Duncan, Numerical Ranges of Operators on Normed Spaces and Elements of Normed Algebras, Cambridge University Press, New York, 1971.

4. M.D. Choi, Completely positive linear maps on complex matrices, Linear Algebra Appl. 10 (1975), 285-290.

5. M.D. Choi, M. Giesinger, J.A. Holbrook and D.W. Kribs, Geometry of higher rank numerical ranges, Linear Multilinear Algebra, 56 (2008), 53-64.

6. C. Chorianopoulos, S. Karanasios and P. Psarrakos, A definition of numerical range of rectangular matrices, Linear Multilinear Algebra 51 (2009), 459-475.

7. C. Chorianopoulos, P. Psarrakos, Birkhoff-James approximate orthogonality sets and numerical ranges, Linear Algebra Appl. 434 (2011), 2089-2108.

8. S. Clark, C.K. Li and N.S. Sze, Multiplicative maps preserving the higher rank numerical ranges and radii, Linear Algebra Appl. 432 (2010), 2729-2738.

9. K.E. Gustafson and D.K.M. Rao, Numerical Range: The Field of Values of Linear Operators and Matrices, Springer-Verlage, New York, 1997.

10. R. Horn and C. Johnson, Topics in Matrix Analysis, Cambridg University Press, New York, 1991.

11. D.W. Kribs, R. Laflamme, D. Poulin and M. Lesosky, Operator quantum error correction, Quant. Inf. Comput. 6 (2006), 383-399.

12. C.K. Li, Y.T. Poon and N.S. Sze, Condition for the higher rank numerical range to be non-empty, Linear Multilinear Algebra 57 (2009), 365-368.

13. C.K. Li and L. Rodman, Numerical range of matrix polynomials, SIAM J. Matrix Analysis Appl. 15 (1994), 1256-1265.

14. J.G. Stampfli and J.P. Williams, Growth conditions and the numerical range in a Banach algebra, Tôhoku Math. J. (2) 20 (1968), 417-424.

15. G.W. Stewart and J.Q. Sun, Matrix Perturbation Theory, Academic Press, San Diego, CA, 1991.

${ }^{1}$ Department of Mathematics, Kerman Science and Research Branch, Islamic Azad University, Kerman, Iran.

E-mail address: mzahraei56@yahoo.com

2 Department of Mathematics, Shahid Bahonar University of Kerman, 76169-

14111, KeRMAN, IrAn.

E-mail address: aghamollaei@uk.ac.ir, gh_aghamollaei@yahoo.com 